\title{
THE DISTRIBUTIVE LATTICE FREE PRODUCT AS A SUBLATTICE OF THE ABELIAN \&-GROUP FREE PRODUCT
}

\author{
WAYNE B. POWELL and CONSTANTINE TSINAKIS
}

(Received 6 April 1981; revised 23 December 1981)

Communicated by J. B. Miller

\begin{abstract}
This paper establishes an important link between the class of abelian l-groups and the class of distributive lattices with a distinguished element. This is accomplished by describing the distributive lattice free product of a family of abelian \&-groups as a naturally generated sublattice of their abelian f-group free product.
\end{abstract}

1980 Mathematics subject classification (Amer. Math. Soc.): primary 06 F 20; secondary 06 D 05.

\section{Introduction}

Let $\Delta$ be a class of similar algebras, and let $\left(A_{i} \mid i \in 9\right)$ be a family of members of $\Delta$. The $\Delta$-free product of this family is an algebra $A \in \Delta$ together with a family of monomorphisms $\left(\alpha_{i}: A_{i} \rightarrow A \mid i \in \mathscr{G}\right)$ such that:

(i) $\cup_{i \in g} \alpha_{i}\left(A_{i}\right)$ generates $A$;

(ii) for each $B \in \Delta$ and every family of homomorphisms $\left(\beta_{i}: A_{i} \rightarrow B \mid i \in \mathscr{9}\right)$, there exists a (necessarily) unique homomorphism $\beta: A \rightarrow B$ satisfying $\beta_{i}=\beta \circ \alpha_{i}$ for all $i \in \mathcal{G}$.

Following the usual practice we shall speak of $A$ as the $\Delta$-free product of $\left(A_{i} \mid i \in \mathscr{9}\right)$ and denote it by $\Delta \bar{\cup}_{i \in \mathcal{G}} A_{i}$ or simply $\bar{\cup}_{i \in \mathscr{G}} A_{i}$.

In this paper we consider the classes $\mathscr{D}_{e}$ of all distributive lattices with a distinguished element $e$ and $\hat{U}$ of all abelian lattice ordered groups ( - groups). These are both varieties (that is, closed under products, subalgebras, and homomorphic images), and each member of either class has a one element subalgebra.

Copyright Australian Mathematical Society 1983 
Hence, free products in these classes always exist. (See Grätzer (1979), page 186 or Pierce (1968), page 107 for general existence theorems on free products.) In what follows we shall find it convenient to always identify the algebras $A_{i}$ with their images $\alpha_{i}\left(A_{i}\right)$ in $\bar{\cup}_{i \in 9} A_{i}$ and thus think of them as subalgebras of $\bar{\cup}_{i \in \mathcal{G}} A_{i}$.

A very important problem in the study of l-groups is to understand the interaction between the group structure and the lattice structure of an f-group. It is well known that the lattice of any l-group is distributive (see for example Bigard and others (1977), page 19). Thus it becomes important to consider the behavior of f-groups in $\mathscr{D}_{e}$. A significant step in this direction was taken by Franchello (1978), who showed that the $\mathscr{Q}_{e}$-free product of a family $\left(G_{i} \mid i \in \mathscr{G}\right)$ of members of $E$ - the class of all f-groups--is the sublattice of the $E$-free product of this family generated by $\cup_{i \in 9} G_{i}$.

The main result of this paper is Theorem 3.3 which asserts that the corresponding result for the class $\mathbb{Q}$ of abelian P-groups also holds. As $\mathbb{A}$ is the least non-trivial variety of f-groups this result seems significant and somewhat surprising.

The proof of Theorem 3.3 depends heavily on a characterization of $D_{e}$-free products (Theorem 2.4) and on a representation for $\mathcal{Q}$-free products (Theorem 3.2). This representation is a special case of a general representation theory for Q-free products (see Powell and Tsinakis (1981)). In addition to this paper and Franchello's work (1978), other contributions to the study of free products of f-groups have been made by Holland and Scrimger (1972), and Martinez (1972), (1973).

Most of the terminology of this paper is standard. Background information on ordered groups can be found in Bigard and others (1977), Conrad (1970), and Fuchs (1963). General references for lattice theoretic concepts are Birkhoff (1967), Grätzer (1970), and Balbes and Dwinger (1974), while the notions of universal algebra can be found in Grätzer (1979), Mal'cev (1973), and Pierce (1968).

\section{A characterization of the distributive lattice free product}

In this section we consider the class $D_{e}$ of all distributive lattices $(L, \wedge, \vee, e)$ with a distinguished element (nullary operation) $e$. A $Q_{e}$-homomorphism is a lattice homomorphism which preserves $e$. In Theorem 2.4 we give a characterization of $\mathscr{D}_{e}$-free products which will directly apply to the abelian f-group free products discussed in the next section. This characterization is similar in spirit to a well-known characterization of free products of bounded distributive lattices (see for example Grätzer (1970), Theorem 5, page 131 or Balbes-Dwinger (1974), 
Theorem 1, page 131), and its proof is an adaptation of the proof of this result. It is worth mentioning at this point that the solution of the word problem for free products in $\mathcal{D}_{e}$ established by Franchello (1978), can be easily inferred from Theorem 2.4 .

For $L \in \mathscr{D}_{e}$ and $S \subseteq L,\langle S\rangle$ will denote the subalgebra of $L$ generated by $S$. Note that $\langle\varnothing\rangle=\{e\}$. If $S$ is a finite non-empty subset of a set $T$, then we write $S \subseteq \cdot T$. For the proof of Theorem 2.4 we shall make use of the following three simple lemmas.

LEMMA 2.1. Let $S \subseteq L \in \mathcal{D}_{e}$. Then

$$
\begin{aligned}
\langle S\rangle & =\left\{\underset{i \in \mathscr{G} \in \mathcal{G}}{\vee} t_{i j} \mid t_{i j} \in S \cup\{e\} ; \mathscr{q} \text { and } \mathscr{q} \text { are finite }\right\} \\
& =\left\{\underset{i \in \mathcal{G} \in \mathcal{G}}{\wedge} \vee r_{i j} \mid r_{i j} \in S \cup\{e\} ; \mathscr{q} \text { and } \mathscr{q} \text { are finite }\right\} .
\end{aligned}
$$

Lemma 2.2. Let $L, L^{\prime} \in \mathscr{W}_{e}$ and $e \in S \subseteq L$. A map $f: S \rightarrow L^{\prime}$ such that $f(e)=e$ can be extended to a $\mathscr{Q}_{e}$-homomorphism $\bar{f}:\langle S\rangle \rightarrow L^{\prime}$ if and only if

$$
\widehat{\wedge}_{i \in \mathcal{G}} t_{i} \leqslant \underset{j \in \mathcal{G}}{\vee} r_{j} \text { implies } \widehat{\wedge}_{i \in \mathcal{G}} f\left(t_{i}\right) \leqslant \underset{j \in \mathscr{G}}{\vee} f\left(r_{j}\right)
$$

whenever $\left\{t_{i} \mid i \in \mathscr{G}\right\},\left\{r_{j} \mid j \in \mathcal{G}\right\} \subseteq S$.

Proof. See for example Balbes and Dwinger (1974), Theorem 1, page 86.

LEMMA 2.3. If $x_{1}, x_{2}, y_{1}, y_{2}$ and $z$ are elements of any distributive lattice such that $x_{1} \vee z \leqslant x_{2} \vee z$ and $y_{1} \wedge z \leqslant y_{2} \wedge z$, then $x_{1} \wedge y_{1} \leqslant x_{2} \vee y_{2}$.

Now let $L \in \mathcal{D}_{e}$. We adopt the following notation.

$$
L^{+}=\{x \in L \mid x \geqslant e\}, \quad L^{-}=\{x \in L \mid x \leqslant e\} .
$$

THEOREM 2.4. Let $L \in \mathbb{D}_{e}$, and let $\left(L_{i} \mid i \in 9\right)$ be a family in $\mathscr{Q}_{e}$ such that each $L_{i}$ is a subalgebra of $L$. Then $L$ is the $\mathbb{O Q}_{e}$-free product of this family if and only if the following conditions are satisfied.

(1) $\left\langle\cup_{i \in \Psi} L_{i}\right\rangle=L$.

(2) For all $\mathcal{G}, \mathfrak{h} \subset .9$ and all families $\left(a_{j} \mid j \in \mathcal{G}\right),\left(b_{k} \mid k \in \mathfrak{h}\right)$ in $L$ such that either $a_{j} \in L_{j}^{+} \backslash\{e\}$ and $b_{k} \in L_{k}^{+}$for all $j \in \mathscr{G}, k \in \mathfrak{H}_{j}$ or $a_{j} \in L_{j}^{-}$and $b_{k} \in$ $L_{k}^{-} \backslash\{e\}$ for all $j \in \mathcal{G}, k \in \mathcal{H}$, the relation

$$
\bigwedge_{j \in \mathfrak{j}} a_{j} \leqslant \underset{k \in \mathcal{h}}{\vee} b_{k}
$$

implies the existence of $i \in \mathfrak{T} \cap$ 政 with $a_{i} \leqslant b_{i}$. 
Proof. (i) Suppose first that $L$ is the free product of $\left(L_{i} \mid i \in 9\right.$ ). Condition (1) holds by the definition of the free product. To establish condition (2), let $\mathscr{f}, \mathcal{K} \subseteq \cdot \mathscr{q}$ indexing the sets $\left\{a_{j} \in L_{j}^{+} \backslash\{e\} \mid j \in \mathscr{G}\right\}$ and $\left\{b_{k} \in L_{k}^{+} \mid k \in \mathscr{K}\right\}$. Suppose that $\wedge_{j \in \mathcal{f}} a_{j} \leqslant V_{k \in \mathcal{r}} b_{k}$ and that the conclusion of (2) is not satisfied. Denote by $C$ the two element chain $\{0,1\}$ with $e_{C}=0$.

If $\mathscr{G} \cap \mathcal{K} \neq \varnothing$, then by assumption $a_{i} \neq b_{i}$ for all $i \in \mathscr{G} \cap \mathcal{K}$. Thus, for each such $i$ there exists a lattice homomorphism $f_{i}: L_{i} \rightarrow C$ such that $f_{i}\left(a_{i}\right)=1$ and $f_{i}\left(b_{i}\right)=0$. Note that $f_{i}$ is actually a $\mathscr{D}_{e}$-homomorphism since $f_{i}(e) \leqslant f_{i}\left(b_{i}\right)=0=$ $e_{C}$.

If $i \in \mathscr{F} \backslash \mathscr{K}$, then $a_{i}>e$, so again there exists a $\mathscr{Q}_{e}$-homomorphism $f_{i}: L_{i} \rightarrow C$ such that $f_{i}\left(a_{i}\right)=1$. Finally, for $i \in \mathscr{G} \backslash \mathcal{G}$, let $f_{i}: L_{i} \rightarrow C$ be the zero map.

By the universal property of the free product, there is a $D_{e}$-homomorphism $f$ : $L \rightarrow C$ such that $f \mid L_{i}=f_{i}$ for all $i \in \mathscr{G}$. But then $1=\wedge_{j \in g} f_{j}\left(a_{j}\right)=\wedge_{j \in q} f\left(a_{j}\right)$ $=f\left(\wedge_{j \in \mathfrak{g}} a_{j}\right)=f\left(\vee_{k \in \mathcal{K}} b_{k}\right)=\vee_{k \in \mathscr{K}} f_{k}\left(b_{k}\right)=0$. This contradiction implies that the conclusion of (2) must indeed be satisfied.

A similar proof takes care of the other case for condition (2).

(ii) Conversely, suppose conditions (1) and (2) are satisfied, and consider a family $\left(f_{i}: L_{i} \rightarrow L^{\prime} \mid i \in 9\right.$ ) of $\mathscr{Q}_{e}$-homomorphisms into $L^{\prime} \in \mathscr{Q}_{e}$. We first verify that the following implication holds.

If $\mathcal{G}, \mathscr{K} \subseteq \cdot \mathscr{G}, a_{j} \in L_{j}$ for each $j \in \mathscr{q}, b_{k} \in L_{k}$ for each $k \in \mathscr{K}$, and

$$
\bigwedge_{j \in \mathfrak{j}} a_{j} \leqslant \underset{k \in \mathfrak{K}}{\vee} b_{k}
$$

in $L$, then $\wedge_{j \in q} f_{j}\left(a_{j}\right) \leqslant \bigvee_{k \in \varsubsetneqq} f_{k}\left(b_{k}\right)$.

For $a_{j}, b_{j}$ as in $(*)$ we get

$$
\bigwedge_{j \in \mathcal{G}}\left(a_{j} \vee e\right) \leqslant \underset{k \in \mathcal{K}}{\vee}\left(b_{k} \vee e\right) \text { and } \bigwedge_{j \in \mathcal{G}}\left(a_{j} \wedge e\right) \leqslant \underset{k \in \mathcal{K}}{\vee}\left(b_{k} \wedge e\right) .
$$

There are four cases which must be considered.

Case 1 . For all $j \in \mathcal{G}, k \in \mathcal{K}, a_{j} \vee e>e$ and $b_{k} \wedge e<e$.

Case II. For all $j \in \mathcal{G}, a_{j} \vee e>e$, and there is $k \in \mathcal{H}$ such that $b_{k} \wedge e=e$.

Case III. There is $j \in \mathscr{q}$ such that $a_{j} \vee e=e$, and for all $k \in \mathcal{K}, b_{k} \wedge e<e$.

Case IV. There are $j \in \mathcal{G}, k \in \mathcal{H}$ such that $a_{j} \vee e=e$ and $b_{k} \wedge e=e$.

We first consider case I. In view of condition (2), there exist $i_{1}, i_{2} \in \mathfrak{q} \cap \mathscr{q}$ such that

$$
a_{i_{1}} \vee e \leqslant b_{i_{1}} \vee e \text { and } a_{i_{2}} \wedge e \leqslant b_{i_{2}} \wedge e
$$


As each $f_{i}$ is a $D_{e}$-homomorphism, it follows that $f_{i_{1}}\left(a_{i_{1}}\right) \vee e \leqslant f_{i_{1}}\left(b_{i_{1}}\right) \vee e$ and $f_{i_{2}}\left(a_{i_{2}}\right) \wedge e \leqslant f_{i_{2}}\left(b_{i_{2}}\right) \wedge e$. Now Lemma 2.3 yields

$$
f_{i_{1}}\left(a_{i_{1}}\right) \wedge f_{i_{2}}\left(a_{i_{2}}\right) \leqslant f_{i_{1}}\left(b_{i_{1}}\right) \vee f_{i_{2}}\left(b_{i_{2}}\right)
$$

which clearly implies the conclusion in $(*)$.

We next consider case II. By condition (2), there exists $i_{1} \in \mathscr{I} \cap \mathcal{f}$ such that $a_{i_{1}} \vee e \leqslant b_{i_{1}} \vee e$. Again this implies that $f_{i_{1}}\left(a_{i_{1}}\right) \vee e \leqslant f_{i_{1}}\left(b_{i_{1}}\right) \vee e$. Now let $k \in \mathscr{F}$ be such that $b_{k} \wedge e=e$, and consider an arbitrary $j \in \mathscr{f}_{\mathcal{H}}$. Then $a_{j} \wedge e \leqslant e \leqslant b_{k} \wedge$ $e$ and hence $f_{j}\left(a_{j}\right) \wedge e \leqslant f_{j}(e)=e=f_{k}(e) \leqslant f_{k}\left(b_{k}\right) \wedge e$. Invoking Lemma 2.3 again we get

$$
f_{i_{1}}\left(a_{i_{1}}\right) \wedge f_{j}\left(a_{j}\right) \leqslant f_{i_{1}}\left(b_{i_{1}}\right) \vee f_{k}\left(b_{k}\right) .
$$

This inequality implies the conclusion in $(*)$.

Case III is treated as case II, whereas case IV immediately implies the conclusion in $(*)$.

Now define $f: \cup_{i \in 9} L_{i} \rightarrow L^{\prime}$ by $f\left(a_{i}\right)=f_{i}\left(a_{i}\right)$ for each $a_{i} \in L_{i}$. In view of condition (1), (*), and Lemma $2.2, f$ can be extended to a $\mathscr{D}_{e}$-homomorphism $\bar{f}$ : $L \rightarrow L^{\prime}$. Evidently $\left.\tilde{f}\right|_{L_{i}}=f_{i}$ for each $i \in \mathscr{G}$, and thus $L$ is the $\mathscr{D}_{e}$-free product of the family $\left(L_{i} \mid i \in 9\right)$.

\section{Distributive lattice free products as sublattices of abelian f-groups}

In this section we show the relationship between the $0_{e}$-free product and the $(\mathcal{A}$-free product of a family of abelian \&-groups (Theorem 3.3). This is accomplished with the use of Theorem 2.4 and the construction of the $\mathbb{Q}$-free product given in Powell and Tsinakis (1981). (See Theorem 3.2 below.)

We write all f-groups additively without regard to commutativity or lack of it. Whenever we view an f-group $G$ as a member of the class $\mathscr{D}_{e}$ we shall always assume that 0 is the distinguished element of $G$. By the direct sum $\oplus_{i \in 4} G_{i}$ of a family $\left(G_{i} \mid i \in \mathscr{9}\right)$ of f-groups we mean the group theoretic sum without regard to order. The cardinal order on a direct sum of l-groups is the componentwise order. We use $\Pi_{i \in 9} G_{i}$ to denote the product of a family $\left(G_{i} \mid i \in \mathscr{9}\right)$ of f-groups. The group and lattice operations on $\Pi_{i \in \varphi} G_{i}$ are defined componentwise.

A convex f-subgroup $P$ of an abelian f-group $G$ is prime (see for example Bigard and others (1977), page 44) if $G / P$ is totally ordered. If $P$ is the collection of all prime subgroups of $G$, then there is a natural embedding $G \rightarrow \prod_{P \in \text { i. }} G / P$ defined by $g \mapsto(\ldots, g+P, \ldots)$. Hence, if $a \neq b$ in $G$, then there is $P \in \mathcal{P}$ such that $b+P<a+P$ in $G / P$.

We start with an immediate consequence of Theorem 2.4 . 
Proposition 3.1. Let $G$ be an f-group (not necessarily abelian) and let $\left(G_{i} \mid i \in \varphi\right.$ ) be a family of l-subgroups of $G$ such that $\cup_{i \in 9} G_{i}$ generates $G$ as an f-group. Let $L=\left\langle\cup_{i \in 9} G_{i}\right\rangle$ be the sublattice of $G$ generated by $\cup_{i \in \mathcal{G}} G_{i}$. The following are equivalent.

(1) $L$ is the $\mathbb{Q}_{e}$-free product of $\left(G_{i} \mid i \in \mathscr{G}\right)$.

(2) For $\mathscr{G}, \mathscr{K} \subseteq \mathscr{G}, a_{j} \in G_{j}^{+} \backslash\{0\}$ for all $j \in \mathscr{F}$, and $b_{k} \in G_{k}^{+}$for all $k \in \mathcal{S}$, the relation

$$
\bigwedge_{j \in \mathcal{G}} a_{j} \leqslant \underset{k \in \mathcal{H}}{\vee} b_{k}
$$

implies the existence of $i \in \mathscr{G} \cap \mathscr{g}$ with $a_{i} \leqslant b_{i}$.

Now, let $\left(G_{i} \mid i \in \mathscr{9}\right)$ be a family of abelian l-groups and let $G={ }^{a} \bar{\cup}_{i \in \mathscr{G}} G_{i}$ be the $\mathbb{Q}$-free product of this family. Consider the set

$$
\Lambda=\left\{(K, T) \mid K=\oplus\left(G_{i} / P_{i}\right) \text {, each } P_{i} \text { is a prime subgroup of } G_{i} \text {, and } T\right. \text { is }
$$
the positive cone of a total order on $K$ extending the cardinal order .

Set $H=\oplus_{i \in q} G_{i}$, and let

$$
\delta: H \rightarrow \prod_{\Lambda}(K, T)
$$

be the diagonal map. That is, if $h=\sum_{i \in 9} h_{i} \in H=\bigoplus_{i \in 9} G_{i}$ and $(K, T)=$ $\left(\oplus_{i \in g}\left(G_{i} / P_{i}\right), T\right) \in \Lambda$, then the $(K, T)$ component of $\delta(H)$ is the element $\sum_{i \in \mathcal{G}}\left(h_{i}+P_{i}\right)$.

With this notation we have the following result established in Powell and Tsinakis (1981).

THEOREM 3.2. $G={ }^{\Theta} \bar{\cup}_{i \in \mathcal{G}} G_{i}$ is the sublattice of $\Pi_{\Lambda}(K, T)$ generated by $\{\delta(h) \mid h$ $\in H\}$.

Note that in view of Theorem 3.2 and Lemma 2.1, every element of $G$ is of the form

$$
g=\underset{j \in \mathfrak{j} \in \mathbb{h}}{\vee} \delta\left(h_{j k}\right)
$$

where $\left(h_{j k} \mid j \in \mathscr{f}, k \in \mathcal{h}\right)$ is a finite family of elements of $H$ and the joins and meets are taken in the product $\Pi_{\Lambda}(K, T)$.

One final bit of terminology: Let $A$ be a torsion free abelian partially ordered group with positive cone $A^{+}$. Call a non-empty subset $S$ of $A$ positively independent provided that whenever $\left\{s_{1}, \ldots, s_{k}\right\} \subseteq S \backslash\{0\},\left\{\lambda_{1}, \ldots, \lambda_{k}\right\} \subseteq \cdot \mathbf{Z}^{+}$. and $\sum_{j=1}^{k} \lambda_{j} s_{j} \in-A^{+}$, then $\lambda_{1}=\cdots=\lambda_{k}=0$ (see Bernau (1969)). Note that $S$ is positively independent if and only if there exists a total order on $A$ with positive 
cone $T \supseteq A^{+} \cup S$. Indeed, if $S$ is positively independent, then $P=\left\{\sum_{j=1}^{n} \lambda_{j} s_{j}+a\right\}$ $\left.\lambda_{j} \in \mathbf{Z}^{+}, S_{j} \in S, a \in A^{+}\right\}$is the positive cone of a partial order on $A$ and $P \supseteq A^{+} \cup S$. But $P$ can be extended to a positive cone $T \supseteq P$ of a total order on $A$ (see for example Fuchs (1973), page 39). The other implication is clear.

We are now prepared to establish the main result of this paper.

THEOREM 3.3. Suppose $\left(G_{i} \mid i \in \mathcal{G}\right)$ is a family of abelian bgroups, and let $G={ }^{Q} \bar{\cup}_{i \in q} G_{i}$ be their $Q$-free product. Then $L=\left\langle\cup_{i \in G} G_{i}\right\rangle$, the sublattice of $G$ generated by $\cup_{i \in \mathcal{G}} G_{i}$, is the $\mathscr{D}_{e}$-free product of the family $\left(G_{i} \mid i \in \mathscr{9}\right)$.

Proof. We will verify that condition (2) of Proposition 3.1 is satisfied by $L$. To this end, let $q, \mathfrak{T}^{\circ} \subseteq 9, a_{j} \in G_{j}^{+} \backslash\{0\}$ for all $j \in \mathcal{G}$, and $b_{k} \in G_{k}^{+}$for all $k \in \mathfrak{h}$. Suppose

$$
\wedge_{j \in \mathfrak{j}} a_{j} \leqslant \underset{k \in \mathcal{K}}{\vee} b_{k}
$$

We need to show that there is $i \in \mathcal{G} \cap \mathcal{K}$ such that $a_{i} \leqslant b_{i}$. Note that $\bar{\cup}_{\ell \in \mathcal{G}} G_{\ell}$ is an f-subgroup of $\bar{U}_{i \in g} G_{i}$, and hence we may assume, without loss of generality, that $4=q \cup \mathfrak{h}$. We consider several cases.

Case I. $\& \cap h=\varnothing$. For each $j \in \mathscr{f}$ let $P_{j}$ be a prime subgroup of $G_{j}$ such that $a_{j} \notin P_{j}$, and for each $k \in \mathcal{H}$ let $P_{k}$ be a prime subgroup of $G_{k}$ such that $b_{k} \in P_{k}$ (for example, let $P_{k}=G_{k}$ ). Note that for each $j \in \mathscr{G}$ and $k \in \mathfrak{K}, a_{j}+P_{j}>P_{j}$ in $G_{j} / P_{j}$ and $b_{k}+P_{k}=P_{k}$ in $G_{k} / P_{k}$. Let now $T$ be the positive cone of any total order on $\bigoplus_{i \in \Psi}\left(G_{i} / P_{i}\right)$ extending the cardinal order. Then

$$
\wedge_{j \in i}\left(a_{j}+P_{j}\right)>0 \text { and } \underset{k \in \mathcal{K}}{\vee}\left(b_{k}+P_{k}\right)=0 \quad \text { in }\left(\bigoplus_{i \in \mathcal{G}}\left(G_{i} / P_{i}\right), T\right) \text {. }
$$

As $\left(\bigoplus_{i \in \mathcal{G}}\left(G_{i} / P_{i}\right), T\right) \in \Lambda$, this contradicts (**).

Case II. $g \cap \mathcal{h}=\left\{i_{1}\right\}$. Suppose $a_{i_{1}} \neq b_{i_{i}}$. Then there is a prime subgroup $P_{i_{1}}$ of $G_{i_{1}}$ such that $a_{i_{1}}+P_{i_{1}}>b_{i_{1}}+P_{i_{1}}$ in $G_{i_{1}} / P_{i_{1}}$. For $j \in \mathscr{G} \backslash\left\{i_{1}\right\}, k \in \mathscr{K} \backslash\left\{i_{1}\right\}$, pick $P_{j}$ and $P_{k}$ as in case 1 . Now let $T$ be the positive cone of a lexicographic order on $\oplus_{i \in 4}\left(G_{i} / P_{i}\right)$ such that $G_{j} / P_{j}$ dominates $G_{i_{1}} / P_{i_{1}}$ for each $j \in \mathcal{g}-\left\{i_{1}\right\}$, and $G_{i_{1}} / P_{i_{1}}$ dominates $G_{k} / P_{k}$ for each $k \in \mathcal{K}-\left\{i_{1}\right\}$. Then $\left(\oplus_{i \in q}\left(G_{i} / P_{i}\right), T\right) \in \Lambda$ while

$$
\wedge\left(a_{j}+P_{j}\right)=a_{i_{1}}+P_{i_{1}}>b_{i_{1}}+P_{i_{1}}=\underset{k \in i}{\vee}\left(b_{k}+P_{k}\right)
$$

on this component. This again contradicts $(* *)$.

Case III. If $\cap h=\left\{i_{1}, i_{2}\right\}$. Suppose $a_{i_{1}} \neq b_{i_{1}}$ and $a_{i_{2}} \neq b_{i_{2}}$. Choose prime subgroups $P_{i_{1}}$ of $G_{i_{1}}$ and $P_{i_{2}}$ of $G_{i_{2}}$ such that $a_{i_{1}}+P_{i_{1}}>b_{i_{1}}+P_{i_{1}}$ and $a_{i_{2}}+P_{i_{2}}>$ $b_{i_{2}}+P_{i_{2}}$. We note that

$$
\left\{\left(a_{i_{1}}+P_{i_{1}}\right)-\left(b_{i_{2}}+P_{i_{2}}\right), \quad\left(a_{i_{2}}+P_{i_{2}}\right)-\left(b_{i_{3}}+P_{i_{1}}\right)\right\}
$$


is a positively independent subset of the cardinal sum of $\left(G_{i_{1}} / P_{i_{1}}\right)$ and $\left(G_{i_{2}} / P_{i_{2}}\right)$. Indeed, if $\lambda, \mu \in \mathbf{Z}^{+}$and

$$
\lambda\left(\left(a_{i_{1}}+P_{i_{1}}\right)-\left(b_{i_{2}}+P_{i_{2}}\right)\right)+\mu\left(\left(a_{i_{2}}+P_{i_{2}}\right)-\left(b_{i_{1}}+P_{i_{1}}\right)\right) \leqslant 0,
$$

then $\left(\lambda a_{i_{1}}-\mu b_{i_{1}}\right)+P_{i_{1}} \leqslant\left(\lambda b_{i_{2}}-\mu a_{i_{2}}\right)+P_{i_{2}}$. As these two elements are disjoint, it follows that

$$
\lambda a_{i_{1}}+P_{i_{1}} \leqslant \mu b_{i_{1}}+P_{i_{1}} \text { and } \mu a_{i_{2}}+P_{i_{2}} \leqslant \lambda b_{i_{2}}+P_{i_{2}} .
$$

Thus $\lambda=\mu=0$, as was to be shown. Hence there is a total order on $\left(G_{i_{1}} / P_{i_{1}}\right) \oplus$ $\left(G_{i_{2}} / P_{i_{2}}\right)$, with positive cone $T^{\prime}$, extending the cardinal order such that $a_{i_{1}}+P_{i_{1}}$, $a_{i_{2}}+P_{i_{2}}>b_{i_{1}}+P_{i_{1}}, b_{i_{2}}+P_{i_{2}}$. For $j \in \mathcal{G}-\left\{i_{1}, i_{2}\right\}, k \in \mathbb{K}-\left\{i_{1}, i_{2}\right\}$, pick $P_{j}$ and $P_{k}$ as in case I. Now define a total order on $\bigoplus_{i \in g}\left(G_{i} / P_{i}\right)$ by lexing the l-groups $G_{j} / P_{j}\left(j \in g-\left\{i_{1}, i_{2}\right\}\right)$ on top, then $\left(\left(G_{i_{1}} / P_{i_{1}}\right) \oplus\left(G_{i_{2}} / P_{i_{2}}\right), T^{\prime}\right)$, and finally the b-groups $G_{k} / P_{k}\left(k \in \mathcal{K}-\left\{i_{1}, i_{2}\right\}\right)$ on the bottom. If $T$ is the positive cone of this order it is clear that $\left(\bigoplus_{i \in \mathcal{G}}\left(G_{i} / P_{i}\right), T\right) \in \Lambda$. Furthermore, $\wedge_{j \in \mathcal{Y}}\left(a_{j}+P_{j}\right) \geqslant\left(a_{i}\right.$ $\left.+P_{i_{1}}\right) \wedge\left(a_{i_{2}}+P_{i_{2}}\right)>\left(b_{i_{1}}+P_{i_{1}}\right) \vee\left(b_{i_{2}}+P_{i_{2}}\right) \geqslant V_{k \in \mathcal{K}}\left(b_{k}+P_{k}\right)$, which again contradicts $(* *)$.

Case IV. $\mathscr{f} \cap \mathcal{K}=\left\{i_{1}, \ldots, i_{n}\right\}, n \geqslant 2$. Suppose there is an element of $\mathscr{q} \cap \mathcal{K}$, say $i_{1}$, such that $a_{i_{1}} \$ b_{i_{1}}$. By the associative law for free products (see for example Mal'cev (1973), page 239) $G=G_{i_{1}} \bar{\cup}\left(\bar{\cup}_{i \neq i_{1}} G_{i}\right)$. Note also that $\bar{\cup}_{i \neq i_{1}} G_{i}$ is simply the R-subgroup of $G$ generated by $\cup_{i \neq i_{1}} G_{i}$. Consider the elements

$$
a=\bigwedge_{i \neq i_{1}} a_{i} \text { and } b=\underset{i \neq i_{1}}{\vee} b_{i} \text { in } \bar{\cup}_{i \neq i_{1}} G_{i} .
$$

We have $a_{i} \wedge a \leqslant b_{i} \vee b$ and hence, by case III, $a \leqslant b$. An easy inductive argument implies that there is $k \in\{2, \ldots, n\}$ such that $a_{i_{k}} \leqslant b_{i_{k}}$.

\section{Final comments and open questions}

In light of Theorem 3.3 and Franchello's result [loc. cit.], the following problem seems interesting.

Problem 4.1. Let $\Delta$ be an arbitrary variety of Rgroups, $\left(G_{i} \mid i \in \mathscr{9}\right)$ a family in $\Delta$, and $G$ the $\Delta$-free product of this family. Is the $\mathscr{D}_{e}$-free product of $\left(G_{i} \mid i \in \mathfrak{G}\right)$ the sublattice of $G$ generated by $\cup_{i \in \mathscr{G}} G_{i}$ ?

It should be noted that if $\Delta$ is an arbitrary variety of f-groups and $\left(G_{i} \mid i \in \mathscr{Y}\right)$ is a family of abelian f-groups, then Problem 4.1 can be answered affirmatively.

Now let $\left(G_{i} \mid i \in 9\right)$ be a family of abelian f-groups and $G={ }^{\mathbb{A}} \bar{\cup}_{i \in \mathscr{Y}} G_{i}$ their $Q$-free product. We have observed that every element of $G$ can be written in the form

$$
g=\underset{j \in j}{\vee} \wedge h_{j \in i k},
$$


where $\left(h_{j k} \mid j \in \mathscr{G}, k \in \mathfrak{K}\right)$ is a finite collection of elements of $H=\bigoplus_{i \in 9} G_{i}$. Such a representation is clearly not unique. Therefore, the question arises as to whether or not we can decide when two such expressions represent the same element of $G$.

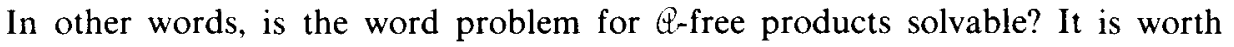
mentioning that a straightforward modification of condition (2) in Proposition 3.1 will not suffice. Take for example the $\mathbb{Q}$-free product of two copies of $\mathbf{Z}$, and consider the positive elements $(1,3),(2,2)$, and $(3,1)$ in this free product. We have $(2,2)<(1,3) \vee(3,1)$, but $(2,2) \$(1,3)$ and $(2,2) \$(3,1)$. Thus, in the condition mentioned, the elements of the $G_{i}(=\mathbf{Z})$ cannot be replaced with arbitrary elements of $H=\mathbf{Z} \oplus \mathbf{Z}$.

Problem 4.2. Is the word problem for $Q$-free products solvable?

\section{References}

R. Balbes and Ph. Dwinger (1974), Distributive lattices (University of Missouri Press, Columbia).

S. Bernau (1969). 'Free abelian lattice groups', Math. Ann. 180, 48-59.

A. Bigard, K. Keimel and S. Wolfenstein (1977), Groupes et anneaux réticulés (Springer-Verlag, New York. Heidelberg, Berlin).

G. Birkhoff (1967), Lattice theory, 3rd ed. (Amer. Math. Soc. Colloq. Publ. 25, Providence).

P. Conrad (1970), Lattice ordered groups (Tulane University. New Orleans).

J. D. Franchello (1978), 'Sublattices of free products of lattice ordered groups', Algebra Universalis 8. $101-110$.

L. Fuchs (1963), Partially ordered algebraic ststems (Pergamon Press, Oxford).

G. Grätzer (1970), Lattice theon, first concepts and distributive lattices (Freeman, San Francisco).

G. Grätzer (1979), Unicersal algebra, 2nd ed. (Springer-Verlag, New York, Heidelberg, Berlin).

W. C. Holland and E. Scrimger (1972), 'Free products of lattice ordered groups', Algebra Universalis 2, 247-254.

A. I. Mal'cev (1973), Algebraic systemis (Springer-Verlag, New York, Heidelberg, Berlin).

J. Martinez (1972), 'Free products in varieties of lattice ordered groups', Czechoslowak Math. J. 22(97), 535-553.

J. Martinez (1973), 'Free products of abelian f-groups', Czechoslowak Math. J. 23(98), 349-361.

R. S. Pierce (1968), Introduction to the theory of abstract algebras (Holt, Rinehart, and Winston, New York).

W. B. Powell and C. Tsinakis (1981), 'Free products in the class of abelian lattice ordered groups', preprint.

Oklahoma State University

Stillwater, Oklahoma 74078

U.S.A.
Vanderbilt University

Nashville. Tennessee

U.S.A. 